\title{
THz sources based on frequency conversion of multi-line molecular lasers in nonlinear crystals and on optically pumped molecular lasers
}

\author{
A. A. Ionin, I. O. Kinyaevskiy, Yu. M. Klimachev, A. A. Kotkov, A. M. Sagitova \\ P.N. Lebedev Physical Institute of the Russian Academy of Sciences, Moscow, Russia, umk@sci.lebedev.ru
}

At present, the main method for generating terahertz radiation is down-conversion of laser radiation in nonlinear crystals. However, at converting the radiation frequency from the visible or near infrared to the terahertz range, the efficiency of the process is limited by the Manley-Row ratio, i.e. the ratio of the photon energies of the converted and incident light on the crystal. Therefore, for applications that require high power of terahertz radiation, the use of a $\mathrm{CO}_{2}$ laser operating at wavelength of $10 \mu \mathrm{m}$ is more preferable than using solid-state near-IR lasers. At the present time in the scientific literature one can find several papers on the conversion of the frequency of the $\mathrm{CO}_{2}$ laser radiation in nonlinear crystals to the terahertz range (see, for example, [1-5]). Note that the conversion efficiency of the super high-power $\mathrm{CO}_{2}$ laser system (UCLA, Neptune Laboratory) under collinear interaction in the GaSe crystal turned out to be lower than for the noncollinear interaction in the GaAs crystal [2], that can be explained by a number of factors: the use of GaSe crystals with low optical quality and realized nonoptimal type of interaction. In practice, $\mathrm{CO}_{2}$ lasers with much lower energy are usually used, and the use of non-collinear phase matching becomes less effective in this case (see, for example, [3]). In turn, the implementation of collinear phase matching allows one the use of a longer length of nonlinear crystal and an increase conversion efficiency. Thus, in [4], at crystal length of $47 \mathrm{~mm}$, the average power of pulsed-periodic terahertz radiation reached $0.26 \mathrm{~mW}$ with efficiency of $10^{-4}$, which is three orders of magnitude greater than in [1]. The use of a doped GaSe crystal instead of a pure one, made it possible to increase the conversion efficiency by additional factor of 2 [5]. It should be noted that in papers [1-5] two $\mathrm{CO}_{2}$ lasers or a two-channel $\mathrm{CO}_{2}$ laser with independent resonators on the same active medium were used. Usually, the generation of the $\mathrm{CO}_{2}$ laser radiation occurs only on one spectral line, although a fundamental possibility of generating a $\mathrm{CO}_{2}$ laser on two or more spectral lines has already been demonstrated (see, for example, the review [6]). The use of pulsed laser emission acting synchronously on two or more spectral lines will allow us to realize a collinear three-frequency interaction in our RFBR project, simplify the optical scheme of the terahertz radiation generator, thanks to the automatic spatialtemporal matching of laser pulses, and reduce optical losses due to a decrease in the number of used elements, that increase the reliability of the entire system. Such a variant of the optical scheme was realized, for example, in [1] mentioned above. However, it should be emphasized that it will be necessary to solve a set of problems in order to ensure real synchronism of the generation of radiation pulses on laser spectral lines both in the Q-switched mode and in the of active mode locking. In addition, it will be necessary to investigate the influence of the parameters of the electric-discharge pump, the quality factor of the laser cavity, and the composition of the active medium of the laser on its spectral characteristics.

Other promising sources of terahertz radiation are optically pumped lasers operating on those molecular gases in which the radiating transition is either purely rotational (for diatomic molecules, molecules of the asymmetric and symmetrical top type except $\mathrm{NH}_{3}$ ), or by inversion or inversion-rotational lasers (for $\mathrm{NH}_{3}$ ) [7]. It was on inversion-rotational transitions of $\mathrm{NH}_{3}$ molecule that laser radiation was generated by French colleagues on our project during optical pumping of ammonia by a quantum-cascade laser [8]. Typically, a $\mathrm{CO}_{2}$ laser is used as the pumping source of terahertz gas lasers [7, 9-10] as the most convenient and wellstudied source of radiation. In contrast to a $\mathrm{CO}_{2}$ laser, a molecular CO laser is capable of operating in the mid-IR range almost simultaneously on hundreds of spectral lines in the wavelength range from 2.5 to 8.7 $\mu \mathrm{m}$, which makes it possible to significantly increase the number of excited molecules and, accordingly, to enrich spectrum of radiation in the terahertz range.

Gas Lasers Laboratory of P.N. Lebedev Physical Institute of the Russian Academy of Sciences is one of the leading teams in Russia in the field of research of molecular lasers with electric discharge pumping. Unique wide-aperture pulsed electron beam sustained discharge laser devices with the possibility of cryogenic cooling of the laser medium working on vibrational-rotational transitions of $\mathrm{CO}, \mathrm{CO}_{2}$, and $\mathrm{N}_{2} \mathrm{O}$ molecules and also on isotopically substituted modifications of these molecules have been created and investigated in the laboratory. An investigation was made of $\mathrm{NH}_{3}$ laser with optical pumping by $\mathrm{CO}_{2}$ laser radiation. Compact slab $\mathrm{CO}$ laser system with pumping by a transverse capacitive radio-frequency (RF) discharge with the possibility of cryogenic cooling of electrodes was developed. At such facilities, the mode of generation of a slab CO laser was realized for the first time, both on the fundamental vibrational transitions and on the firstovertone vibrational transitions of the $\mathrm{CO}$ molecule.

Within the framework of this project, it is planned to use laser systems that will be developed on the basis of existing laser devices pumped with an active medium by a pulsed electron beam sustained discharge [11], a pulsed-periodic RF discharge [12] and a DC discharge [13]. In addition, the members of 
the scientific team have experience in the implementation Q-switching modes and of the active mode-locking of molecular $\mathrm{CO}$ [14] and $\mathrm{CO}_{2}$ lasers [15]. Our studies have been carried out on the frequency conversion of molecular gas lasers in various nonlinear crystals, some of which are expected to be used in carrying out work under this project.

The most close to the tasks of the project are the results of generation of the sum and difference frequencies of $\mathrm{CO}$ and $\mathrm{CO}_{2}$ laser emission in nonlinear $\mathrm{GaSe}$ and $\mathrm{AgGaSe}_{2}$ crystals [13, 16], as well as $\mathrm{PbIn}_{6} \mathrm{Te}_{10}$ [17] into wavelength interval from 2.5 to $16.6 \mu \mathrm{m}$. A large number (more than 1000) of the spectral lines of a $\mathrm{CO}$ laser makes it possible to realize single-stage frequency converters by generating the difference frequencies of both bands (fundamental and first-overtone) and covering the long-wave part of the mid-IR band up to $30 \mu \mathrm{m}$. The generation of difference frequencies between closely spaced spectral lines of a $\mathrm{CO}$ laser from one or another radiation band allows one to cover the entire terahertz spectral range [18]. In the paper [19], based on an analysis of the nonlinear optical properties of the new $\mathrm{PbIn}_{6} \mathrm{Te}_{10}$ crystal, the possibility of conversion of radiation on the lines of $\mathrm{CO}$ and $\mathrm{CO}_{2}$ lasers into the spectral interval of wavelengths from 130 to $1000 \mu \mathrm{m}$ (from 0.3 to $2.3 \mathrm{THz}$ ) is shown.

The research was supported by the RFBR (Project No. 18-52-16019)

\section{References}

1. V.V. Bezotosnyi, E.A. Cheshev, M.V. Gorbunkov et al., Coherent $\mathrm{THz}$ repetitive pulse generation in a $\mathrm{GaSe}$ crystal by dual-wavelength Nd:YLF laser // Physics Procedia 72, 405 (2015)

2. S.Ya. Tochitsky, J.E. Ralph, C. Sung, C. Joshi, Generation of megawatt-power terahertz pulses by noncollinear difference-frequency mixing in GaAs // J. of Appl. Phys. 98, 026101 (2005).

3. Y. Lu, X. Wang, L. Miao, et. al., Efficient and widely step-tunable terahertz generation with a dual-wavelenght $\mathrm{CO}_{2}$ laser // Appl. Phys. B 103, 387 (2011).

4. Y. Jiang, Yu.J. Ding, Efficient terahertz generation from two collinearly propagating $\mathrm{CO}_{2}$ laser pulses // Appl. Phys. Lett. 91, 091108 (2007).

5. D.-D. Sie, D. Guo, L.-M. Zhang et al., Frequency conversion of a nanosecond $\mathrm{CO}_{2}$ laser to the $\mathrm{THz}$ range in doped GaSe crystals // Fundamental problems of modern materials science, 9 (4) 486 (2012) (in Rissian).

6. D.J. Biswas, A.K. Nath, U. Nundy, U.K. Chatterjee, Multiline CO2 lasers and their uses // Prog. Quant. Electr. 11, 1 (1990).

7. A.A. Vedenov, G.D. Myl'nikov, D.N. Sobolenko, Generation of coherent far-infrared radiation using lasers // Physics-Uspekhi (Advances in Physical Sciences). 25, 833 (1982).

8. A. Pagies, G. Ducournau, J.-F. Lampin, Lowthreshold terahertz molecular laser optically pumped by a quantum cascade laser // APL Photonics. 1, 031302 (2016).

9. L.F.L. Costa, J.C.S. Moraes, F.C. Cruz, et al., Infrared and far-infrared spectroscopy of ${ }^{13} \mathrm{CH}_{3} \mathrm{OH}$ : TeraHertz laser lines and assignments // Applied Physics B. 86(4) 703 (2007).

10. F.C. Cruz, T. Nogueira, L.F.L. Costa, et al., Continuous and Pulsed THz generation with molecular gas lasers and photoconductive antennas gated by femtosecond pulses // AIP Conference Proceedings 992, 383 (2008).

11. A. Ionin, Yu Klimachev, A. Kotkov, et al., Multiline Laser Probing for Active Media CO:He, CO: $\mathrm{N}_{2}$, and CO: $\mathrm{O}_{2}$ in Wide-Aperture Pulsed Amplifier // Journal of Russian Laser Research. 27(1) 33 (2006).

12. A.A. Ionin, Yu.V. Kochetkov, A.Yu. Kozlov, et al., Q-switched slab RF discharge CO laser // Laser Phys. Lett. 14, 055001 (2017).

13. A. A. Ionin, I. O. Kinyaevskiy, Yu. M. Klimachev, and A. A. Kotkov, Frequency Conversion of Radiation of IR Molecular Gas Lasers in Nonlinear Crystals (A Review) // Optics and Spectroscopy. 119, 356 (2015).

14. O.V. Budilova, A.A. Ionin, I.O. Kinyaevskiy, et al., Mode-locked and Q-switched carbon monoxide laser system // Optics Communications 345, 163 (2015).

15. A.A. Ionin, I.O. Kinyaevskiy, Yu.M. Klimachev et al., $\mathrm{CO}_{2}$ laser with active mode-locking or Q-switching // VI International Scientific Youth School-Conference "Modern Problems of Physics and Technology", Theses of reports, Part 1, M: NRNU MEPhI, 322 (2017). (in Rissian).

16. O.V. Budilova, A.A.Ionin, I.O.Kinyaevskiy, et al., Ultra-broadband hybrid infrared laser system // Optics Communications 363, 26 (2016).

17. A.A. Ionin, I.O. Kinyaevskiy, Yu.M. Klimachev, et al., Frequency conversion of molecular gas lasers in $\mathrm{PbIn}_{6} \mathrm{Te}_{10}$ crystal within mid-IR range // Optics Letters. 41, 2390 (2016).

18. Yu.M. Andreev, A.A. Ionin, I.O. Kinyaevskiy, et al., Frequency doubling and mixing of the radiation of carbon monoxide lasers in nonlinear $\mathrm{ZnGeP}_{2}$ and $\mathrm{GaSe}$ crystals // Journal of Optical Technology. 78, 102 (2011).

19. Yu.M. Andreev, V.V. Badikov, A.A. Ionin, et al., Optical properties of $\mathrm{PbIn}_{6} \mathrm{Te}_{10}$ in the long-wave IR // Laser Physics Letters. 13, 125405 (2016). 